\title{
Hematoma Subcapsular del Hígado. Morfología y Resultados del Tratamiento Quirúrgico
}

\author{
Hepatic Subcapsular Hematoma. Morphology and Results of Surgical Treatment
}

\author{
Carlos Manterola ${ }^{1,2}$
}

\begin{abstract}
MANTEROLA, C. Hematoma subcapsular del hígado. Morfología y resultados del tratamiento quirúrgico. Int. J. Morphol., 39(3):886$889,2021$.

RESUMEN: El hematoma subcapsular del hígado (HSH), es una entidad poco frecuente. La mayoría de casos reportados se asocian al embarazo, en el contexto de síndrome de recuento bajo de plaquetas, elevación de enzimas hepáticas, y hemólisis. Otras patologías relacionadas son ruptura de carcinoma hepatocelular, adenoma, hiperplasia nodular focal; y amilosis. El objetivo de este artículo fue reportar morfología y resultados del tratamiento quirúrgico observados en una serie de pacientes con HSH, en términos de morbilidad postoperatoria (MPO). Serie de casos retrospectiva de pacientes con HSH intervenidos de forma consecutiva en Clínica RedSalud Mayor Temuco, entre 2004 y 2019. La variable resultado fue MPO. Otras variables de interés fueron edad, sexo, etiología, diámetro, necesidad de transfusión y tiempo de hospitalización. Se utilizó estadística descriptiva. Se trató a 7 pacientes (71,4 \% mujeres), con una mediana de edad de 46 años. La mediana del diámetro del HSH, tiempo quirúrgico y estancia hospitalaria fueron $11 \mathrm{~cm}, 105$ min y 5 días, respectivamente. No hubo necesidad de reintervenciones. Con una mediana de seguimiento de 31 meses, no se verificó MPO ni mortalidad. El HSH es una condición poco frecuente, y la evidencia disponible escasa. Puede asociarse a condiciones benignas y malignas. Requiere un alto índice de sospecha para un diagnóstico oportuno.Su pronóstico depende de la etiología.
\end{abstract}

PALABRAS CLAVE: Hematoma subcapsular del hígado; Adenoma hepatocelular; Tumores hepáticos; Hepatectomía.

\section{INTRODUCCIÓN}

El hematoma subcapsular del hígado (HSH), es una condición infrecuente que se asocia a hipertensión del embarazo, como pre-eclampsia y síndrome HELLP (Ben Hamoula et al., 2008); trauma abdominal (Moore \& Moore, 2010), tumores hepáticos primarios benignos como adenomas, hiperplasia nodular focal y hemangiomas (Ji et al., 2000; Dokmak et al., 2009; Huurman \& Schaapherder, 2010); y malignos, como carcinoma hepatocelular (Maoz et $a l ., 2010)$. También se ha reportado HSH en relación con colecistectomía (Guercio et al., 2008; Shibuya et al., 2010), colangiografía endoscópica (Servide et al., 2016; Roldán Villavicenci et al., 2019), colonoscopia (Jammal et al., 2013), litotripsia extracorpórea (Kim et al., 2010; Bi et al., 2018), y litotripsia biliar (McGrath et al., 1990). También hay reportes en los que el origen no fue posible precisar (Maoz et al.; Cabral Matos, 2018).

La rotura de un HSH, se considera una complicación grave, pues implica riesgos de morbilidad y mortalidad.

La AAST-OIS (Asociación Americana para la Cirugía del Trauma), en la clasificación de los traumatismos he- páticos, clasifica al HSH en grados del I al IV; estimándose al grado IV como una lesión compleja, debido al alto riesgo de mortalidad asociada (Moore \& Moore) (Tabla I; Fig. 1).

El objetivo de este artículo fue reportar la morfología y resultados del tratamiento quirúrgico observados en una serie de pacientes con HSH, en términos de morbilidad postoperatoria (MPO).

\section{MATERIAL Y MÉTODO}

Este reporte, fue escrito siguiendo las directrices de la iniciativa MInCir para el reporte de estudios descriptivos observacionales (Manterola \& Otzen, 2017).

\section{Diseño del estudio: Serie retrospectiva de casos.}

Escenario: El estudio se realizó en Clínica RedSalud Mayor Temuco. El período de reclutamiento fue entre enero de 2004 y agosto de 2019 (15 años). 
Tabla I. Clasificación del Trauma Hepático (AAST-OIS).

\begin{tabular}{cl}
\hline Grado & \multicolumn{1}{c}{ Descripción } \\
\hline I & $\begin{array}{l}\text { Hematoma subcapsular }<10 \% \text { de área de superficie no expansivo o laceración capsular sin sangrado }<1 \mathrm{~cm} \text { de } \\
\text { profundidad. }\end{array}$ \\
II & $\begin{array}{l}\text { Hematoma subcapsular que involucra el } 10-50 \% \text { del área de superficie, no expansivo, o laceración capsular con sangrado } \\
\text { activo de } 1-3 \mathrm{~cm} \text { de profundidad. }\end{array}$ \\
III & Hematoma subcapsular $>50 \%$ del área de superficie o laceración $>3 \mathrm{~cm}$ de profundidad. \\
IV & Hematoma parenquimatoso roto con sangrado activo, o laceración, o hematoma parenquimatoso que involucra el $25-75$ \\
\% de un lóbulo hepático o que a fecta $1-3$ segmentos de Couinaud.
\end{tabular}
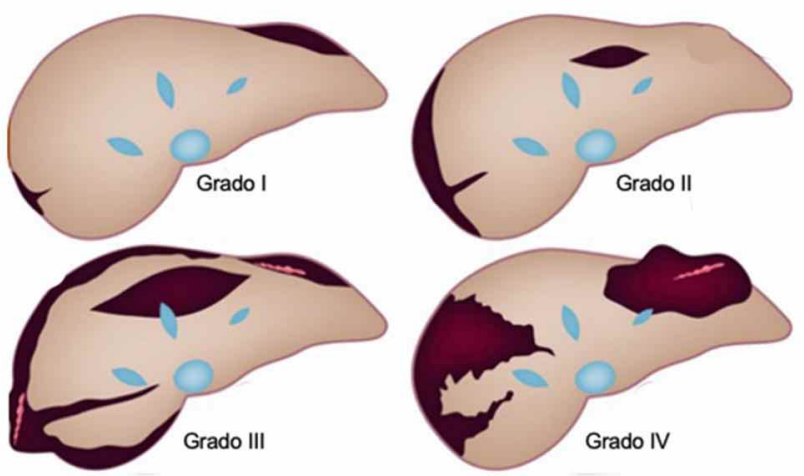

Fig. 1. Morfología del HSH según clasificación de la AAST.

Participantes: Se incluyeron todos los pacientes con $\mathrm{HSH}$, definido por la clasificación AAST-OIS (Moore \& Moore), diagnosticados por tomografía axial computarizada abdominal (Fig. 2), sometidos consecutivamente a cirugía por el autor (CM). No se consideraron criterios de exclusión.

Tamaño de la muestra: Dado que se trata de un estudio descriptivo, no se estimó el tamaño de la muestra. Se consideraron todos los pacientes con HSH sometidos a cirugía en el período e institución indicados.

Variables: La variable resultado fue MPO, la que fue medida hasta 16 meses después de la cirugía, considerándose de forma dicotómica (presente o ausente). Otras variables de interés fueron edad, sexo, etiología, diámetro, tipo de HSH, necesidad de transfusión de glóbulos rojos y tiempo de hospitalización.

Protocolo de seguimiento: Después del alta hospitalaria, los pacientes fueron seguidos con controles clínicos (pruebas generales de laboratorio y tomografía computarizada de abdomen), en los meses 1 y 6 ; y luego una vez al año.

Herramientas estadísticas: La recolección de datos se realizó y analizó con el programa Stata 11.0 / SE®. Se utilizó estadística descriptiva, calculando porcentajes y aplicando medidas de tendencia central y dispersión (medianas y valores mínimos y máximos).

Sesgos: se redujeron con una recogida de datos enmascarada y un seguimiento completo de los pacientes que componen esta serie durante al menos 16 meses (último paciente reclutado).

Ética: Se observaron las pautas éticas para la investigación definidas por la Declaración de Helsinki (Helsinki Statement, 2013). Todos los pacientes dieron su consentimiento informado por escrito.

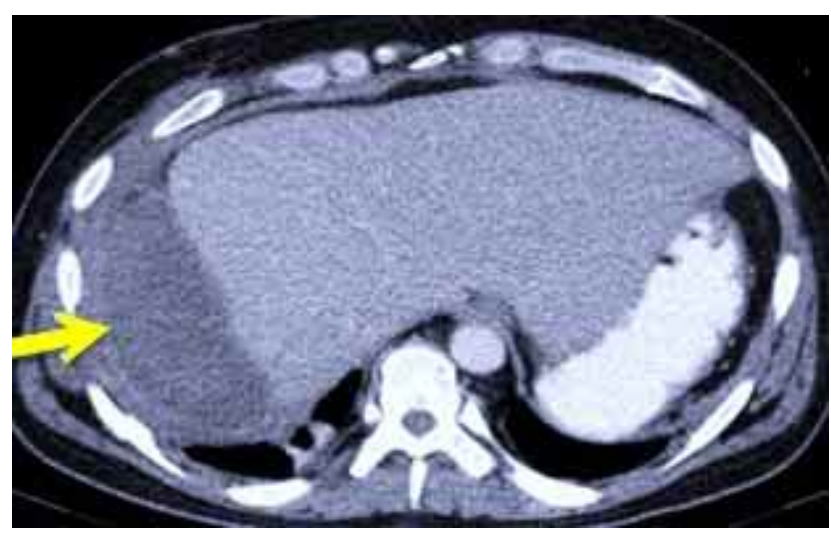

Fig. 2. Imagen tomográfica de un paciente con HSH contenido.

\section{RESULTADOS}

Durante el período de estudio, 7 pacientes fueron operados por HSH; con una mediana de edad de 46 años (71,4 \% sexo femenino). Dos pacientes $(28,6 \%)$, tenían como antecedentes el estar cursando un embarazo (de 15 y 20 semanas respectivamente), sin evidenciarse daño del producto de la concepción (ambas tuvieron partos normales con recién nacidos sin problemas).

La etiología fue predominantemente idiopática. La mediana del diámetro, tiempo quirúrgico, transfusión de 
glóbulos rojos, y tiempo de hospitalización; fue de $11 \mathrm{~cm}$, 105 min, 4 unidades, y 5 días (Tabla I).

La localización fue de predominio en lóbulo derecho $(85,7 \%)$; y el tipo de HSC más común fue el tipo II $(57,1 \%)$ (Tabla II; Fig. 1$)$.

Tabla II. Características clínicas de los pacientes en estudio $(\mathrm{N}=7)$.

\begin{tabular}{lcc}
\hline Variable & Mediana & Mínimo - Máximo \\
\hline Edad (años) & 46 & $28-58$ \\
Diámetro mayor (cm)* & 11 & $8-20$ \\
Hemoglobina (g/dl) & 10 & $8,5-11,5$ \\
Tiempo quirúrgico (min) & 105 & $65-140$ \\
Transfusión de GR & 4 & $2-6$ \\
(unidades) & 5 & $4-7$ \\
Hospitalización (días) & 31 & $16-42$ \\
Seguimiento (meses) & & \\
\hline
\end{tabular}

GR: Glóbulos rojos.

La cirugía realizada en la totalidad de los casos consistió en drenaje y hemostasia. En dos casos (HSH de origen traumático), fue necesario recurrir a empaquetamiento y revisión a las 48 horas, oportunidad en la que retiraron las compresas y se realizó la laparorrafia sin inconvenientes. Luego de ello, no fue necesario reintervenir a ningún paciente.

Con una mediana de seguimiento de 31 meses, no se objetivó MPO ni mortalidad.

\section{DISCUSIÓN}

Novedad de la propuesta: Se trata de una serie de casos consecutivos de $\mathrm{HSH}$, que fue tratada de forma quirúrgica, evolucionando satisfactoriamente.

Comparación con resultados anteriores: El HSH es una emergencia quirúrgica de patogénesis multifactorial poco clara, asociada a una amplia gama de afecciones que comparten características comúnes de deterioro de la integridad del parénquima y su vascularización. La complicación más temida del HSH es la ruptura, pues se asocia a una mortalidad de hasta $75 \%$ (Cabral Matos).

El HSH espontáneo es muy infrecuente, y se ha asociado a síndrome HELLP, definido como asociación de hemólisis, citólisis hepática y trombocitopenia. La fisiopatología del HSH es poco conocida, pero se explicaría por un síndrome microangiopático con hemólisis, asociado a coagulación intravascular diseminada; lo que a nivel hepático conduciría a necrosis y hemorragia espon- tánea que es contenida por la cápsula de Glisson inicialmente, pero que puede romperse (Cabral Matos). Existe evidencia respecto a que la ruptura y la hemorragia (Fig. 3 ), son complicaciones frecuentes en pacientes con adenoma hepatocelular (hasta un $27 \%$ ). Esto puede explicarse por las características del adenoma, que es un tumor altamente vascularizado, con sinusoides de pared delgada, vasos de neoformación y soporte de tejido conectivo escaso; a lo que se ha de agregar que carecen de cápsula tumoral fibrosa (Klompenhouwer et al., 2017).

El cuadro clínico se caracteriza por dolor en cuadrante superior derecho, acompañado de hepatomegalia, acompañado de náuseas, palidez; ocasionalmente disnea, e incluso lipotimia, durante la etapa de formación del HSH. Posteriormente, puede incrementarse el dolor y de forma súbita, desencadenarse shock hipovolémico en casos de ruptura del HSH (Maoz et al.).

El estudio por imágenes suele ser difícil en el contexto de la urgencia del cuadro; pero en general, el ultrasonido puede aportar confirmando una colección líquida hepática, e incluso con la sospecha de una fisura de la cápsula cuando existe derrame intraperitoneal asociado. La tomografía computarizada permite una valoración más fina de la lesión, su localización y extensión. Por otra parte, la arteriografía permite verificar la arteriola rota; y por ende, se puede utilizar como medio para realizar hemostasia mediante la embolización arterial (Tamimi \& Alawad, 2019).

El tratamiento del HSH es complejo y las opciones deben seleccionarse en función de la estabilidad clínica, la causa subyacente y la extensión de la hemorragia, la función hepática y de coagulación. La hemostasia es el objetivo principal, la que se realiza mediante reanimación activa con fluidos, administración de hemoderivados y corrección de la coagulopatía. Es así como, el tratamiento conservador (incluido el control con imágenes seriadas), puede ser apropiado si el paciente está estable y la cápsula hepática intacta (Srinivasa et al., 2015; Tamimi \& Alawad). Sin embargo, en casos de pre-ruptura y ruptura, el tratamiento suele ser quirúrgico. El que consiste en la evacuación completa del hematoma, y hemostasia con medios conservadores como empaquetamiento o aplicación de sustancias hemostáticas (Rosales \& Que, 2016). Si estas maniobras no permiten controlar la hemorragia, se puede recurrir a la ligadura de la arteria hepática o incluso a resecciones hepáticas (Cabral Matos).

Limitaciones: Como limitaciones, se ha de considerar que se trata de una serie de casos pequeña, y que la evidencia existente respecto del HSH es pobre, como para poder comparar resultados. 


\section{CONCLUSIÓN}

Es complejo plantear una conclusión, sobre todo cuando se trata del reporte de una serie de casos pequeña y de una patología poco frecuente. Quizás, más que plantear una conclusión, comentar la lección aprendida respecto de la toma de decisiones terapéuticas.

MANTEROLA, C. Hepatic subcapsular hematoma. Morphology and results of surgical treatment. Int. J. Morphol., 39(3):886-889, 2021.

SUMMARY: Subcapsular hematoma of the liver (SHL) is a rare entity. The majority of cases are associated with pregnancy, in the context of low platelet count syndrome, elevated liver enzymes, and hemolysis. Other related pathologies are ruptured hepatocellular carcinoma, adenoma, focal nodular hyperplasia, and amyloidosis. The aim of this article was to report morphology and results of the surgical treatment observed in a series of patients with SHL, in terms of postoperative morbidity (POM). Retrospective case series of patients with SHL who were intervened consecutively at Clínica RedSalud Mayor Temuco, between 2004 and 2019. The results variable was MPO. Other variables of interest were age, sex, etiology, diameter, need for transfusion, and length of hospitalization. Descriptive statistics were used; 7 patients (71.4 $\%$ women) were treated with a median age of 46 years. The median diameter of SHL, surgical time, and hospital stay were 11 months, no POM and mortality were verified. SHL is a rare condition, and the available evidence is scarce. It can be associated with benign and malignant diseases. It requires a high index of suspicion for a timely diagnosis. Its prognosis depends on the etiology.

KEY WORDS: Hepatic Subcapsular Hematoma; Liver Subcapsular Hematoma; Subcapsular hematoma of the liver; Hepatocellular adenoma; Liver neoplasms; Hepatectomy.

\section{REFERENCIAS BIBLIOGRÁFICAS}

Ben Hamoula, S.; Khoudhair, H.; Fitouhi, L.; Bouguerra, B. \& Sfar, R. Hepatic subcapsular hematoma as a complication of pregnancy. Tunis Med., 86(6):604-6, 2008.

Bi, Y. W.; Wang, D.; Du, T. T.; Liao, Z.; Li, Z. S. \& Hu, L. H. Hepatic subcapsular hematoma breaking into the abdominal cavity after extracorporeal shock wave lithotripsy for pancreatic stones. J. Dig. Dis., 19(5):314-7, 2018.

Cabral Matos, E. Spontaneous hepatic haemorrhage of unknown cause -A case report. Acta Radiol. Port., 30(1):57-62, 2018.

Dokmak, S.; Paradis, V.; Vilgrain, V.; Sauvanet, A.; Farges, O.; Valla, D.; Bedossa, P. \& Belghiti, J. A single-center surgical experience of 122 patients with single and multiple hepatocellular adenomas. Gastroenterology, 137(5):1698-705, 2009.

Guercio, G.; Sandonato, L.; Cintorino, D.; Ricotta, C. \& Diana, G. Hemoperitoneum from rupture of liver subcapsular hematoma after laparoscopic cholecystectomy attributed to ketorolac. Report of a case. G. Chir, 29(8-9):351-3, 2008.
Helsinki Statement. WMA Declaration of Helsinki - Ethical Principles for Medical Research Involving Human Subjects. Fortaleza, 64th WMA General Assembly, 2013. Available from: https://www.wma.net/ policies-post/wma-declaration-of-helsinki-ethical-principles-formedical-research-involving-human-subjects

Huurman, V. A. L. \& Schaapherder, A. F. M. Management of ruptured hepatocellular adenoma. Dig. Surg., 27(1):56-60, 2010.

Jammal, M.; Valesky, W.; Das, D.; Brown, C. \& Kapoor, M. Subcapsular liver hematoma after colonoscopy diagnosed by emergency department bedside ultrasonography. J. Emerg. Med., 45(4):598-601, 2013.

Ji, Y.; Zhu, X.; Sun, H.; Tan, Y.; Ma, Z.; Ye, Q.; Sujie, A. \& Tang, Z. Hepatocellular adenoma and focal nodular hyperplasia: a series of 24 patients with clinicopathological and radiological correlation. Chin. Med. J. (Engl.), 113(9):852-7, 2000.

Kim, T. B.; Park, H. K.; Lee, K. Y.; Kim, K. H.; Jung, H. \& Yoon, S. J. Lifethreatening complication after extracorporeal shock wave lithotripsy for a renal stone: a hepatic subcapsular hematoma. Korean J. Urol., 51(3):212-5, 2010.

Klompenhouwer, A. J.; de Man, R. A.; Thomeer, M. G. J. \& Ijzermans, J. N. M. Management and outcome of hepatocellular adenoma with massive bleeding at presentation. World J. Gastroenterol., 23(25):457986, 2017.

Manterola, C. \& Otzen, T. Checklist for reporting results using observational descriptive studies as research designs. The MInCir initiative. Int. J. Morphol., 35(1):72-76, 2017.

Maoz, D.; Sharon, E.; Chen, Y. \& Grief, F. Spontaneous hepatic rupture: 13-year experience of a single center. Eur. J. Gastroenterol. Hepatol., 22(8):997-1000, 2010.

Moore, E. E. \& Moore, F. A. American Association for the Surgery of Trauma Organ Injury Scaling: 50th anniversary review article of the Journal of Trauma. J. Trauma, 69(6):1600-1, 2010.

Roldán Villavicenci, J. I.; Prieto Calvo, M. \& Mateo, M. G. Post-ERCP hepatic subcapsular hematoma, from conservative therapy to emergency surgery: an unusual though extremely serious complication. Rev. Esp. Enferm. Dig., 111(9):721-3, 2019.

Rosales, A. \& Que, F. G. Spontaneous hepatic hemorrhage: a single institution's 16-year experience. Am. Surg., 82(11):1117-20, 2016.

Servide, M. J.; Prieto, M. \& Marquina, T. Hepatic subcapsular hematoma: a rare late complication after ERCP. Rev. Esp. Enferm. Dig., 108(4):2345, 2016.

Shibuya, K.; Midorikawa, Y.; Mushiake, H.; Watanabe, M.; Yamakawa, T. \& Sugiyama, Y. Ruptured hepatic subcapsular hematoma following laparoscopic cholecystectomy: report of a case. Biosci. Trends, 4(6):3558, 2010.

Srinivasa, S.; Lee, W. G.; Aldameh, A. \& Koea, J. B. Spontaneous hepatic haemorrhage: a review of pathogenesis, aetiology and treatment. $H P B$ (Oxford), 17(10):872-80, 2015.

Tamimi, A. A. \& Alawad, A. A. Large spontaneous subcapsular hematoma of the liver: a rare case report. Pan. Afr. Med. J., 32:16, 2019.

\section{Dirección para correspondencia:}

Dr. Carlos Manterola

Departamento de Cirugía y Traumatología; y

Centro de Estudios Morfológicos y Quirúrgicos - CEMyQ

Universidad de La Frontera

Temuco -CHILE

E-mail: carlos.manterola@ufrontera.cl

Recibido : 26-12-2020

Aceptado: 23-02-2021 